\title{
Marchiafava-Bignami Disease (MBD) in a Non- Alcoholic Patient: A Case Report
}

\author{
Santosh B. Murthy, Ali Jawaid, John E. Bock, Salah U. Qureshi, Paul E. Schulz
}

Can. J. Neurol. Sci. 2010; 37: 138-140

Marchiafava-Bignami disease (MBD) is a rare disease characterized by primary focal demyelination of the corpus callosum, although lesions involving the cerebellar peduncles and the cerebral hemispheres have also been reported. ${ }^{1}$ Marchiafava and Bignami, in 1903, first described the disease in three Italian patents with a history of red wine consumption. ${ }^{2}$ The patients typically present with neuropsychiatric complaints, dysarthria, tetraparesis, astasia-abasia, impaired consciousness and symptoms of inter hemispheric disconnection. Owing to a lack of specific clinical features, the modern diagnosis is almost always based entirely on the magnetic resonance image (MRI) findings. ${ }^{3}$

Marchiafava-Bignami disease has traditionally been described as a consequence of chronic alcohol abuse. However, the occurrence of the disease in non-alcoholics suggests that the precise etiology remains to be established. ${ }^{4-5}$ Here, we describe an unusual presentation of MBD in a non-alcoholic.

\section{Case Report}

A 45-year-old, left-handed, Caucasian, non-alcoholic industrial worker presented with a history of lapses in memory for over a year. His family also noticed changes in his behavior, although he remained oblivious to it. He had become much quieter and socially withdrawn. He had no weakness, numbness or tingling. There was no history of urinary incontinence or gait disturbance. He had an occupational exposure to acetone and methyl ethyl ketone over a period of 14 years.

On neurological examination, the deep tendon reflexes were diminished bilaterally. Frontal release signs (glabellar and snout) were positive. The rest of the general neurologic examination was normal. A neuropsychiatric evaluation revealed marked impairment in immediate recall and episodic memory, language and attention. There were mild deficits on visuospatial and semantic testing.

The basic metabolic panel and vasculitic workup were within normal limits. The MRI brain showed focal demyelination involving the genu and splenium of the corpus callosum (Figures $1,2)$. There was diffusion restriction in the corpus callosum and a corresponding subtle change on the apparent diffusion coefficient map. A lumbar puncture showed a normal glucose, proteins and cell count. The IgG synthesis and oligoclonal bands were within the normal range. A paraneo-plastic panel was unrevealing.

The constellation of clinical symptoms, the radiological findings, and the lack of an alternate diagnosis suggested a diagnosis of probable MBD.

\section{Discussion}

Our patient presented with a one year history of memory impairment with behavioral changes accompanied by changes in the corpus callosum on MRI. His clinical and laboratory findings were consistent with MBD.

Marchiafava-Bignami disease has been reported in about 250 patients from several countries encompassing all races and both genders. Clinically, it can be divided into an acute and chronic phase. The acute phase presents with the sudden onset of impaired consciousness, seizures, dysphasia and diffuse muscular hypertonia. Chronic MBD is characterized by motor impairment (apraxia, dysarthria, gait disturbance) and cognitive impairment (agraphia, alexia, disorientation, memory deficits). ${ }^{2}$ Our patient's presentation was more consistent with the chronic form of MBD as he had cognitive impairment, but his level of consciousness was normal and he had no seizures, dysphasia or hypertonia.

In terms of etiology, a strong association between alcohol consumption and MBD has been observed. However, the exact role of alcohol in the etiology of MBD remains to be defined. ${ }^{2}$ One hypothesis is that a toxin present in alcohol may be responsible for the demyelination of the corpus callosum in MBD, while non-drinkers may be exposed to it from another source. Alternately, it has been suggested that severe liver dysfunction in chronic alcoholism may raise blood ammonia levels, which may lead to encephalopathy, edema and demyelination of the corpus callosum. ${ }^{6}$ Malnutrition has also been suggested in the pathogenesis, but a responsible micronutrient deficiency has not been defined. Vitamin Bcomplex deficiency has also been suggested and has been noticed in patients with a long-term history of alcoholism and malnutrition..$^{7-8}$ Our patient was unusual in lacking a history of both alcoholism and malnutrition. He did however, have an occupational exposure to acetone. In the past, studies have

From the Department of Neurology (SBM, AJ, SUQ, PES), Baylor College of Medicine, Houston; Riverwood Court Suite (JEB), Conroe, Texas, USA. Received February 23, 2009. Final Revisions Submitted July 28, 2009. Correspondence to: Paul E. Schulz, Neurology, Neuroscience \& Translational Biology Mail Stop: NB302, Department of Neurology, Baylor College of Medicine, 6501 Fannin Street, Houston, Texas, 77030, USA. 

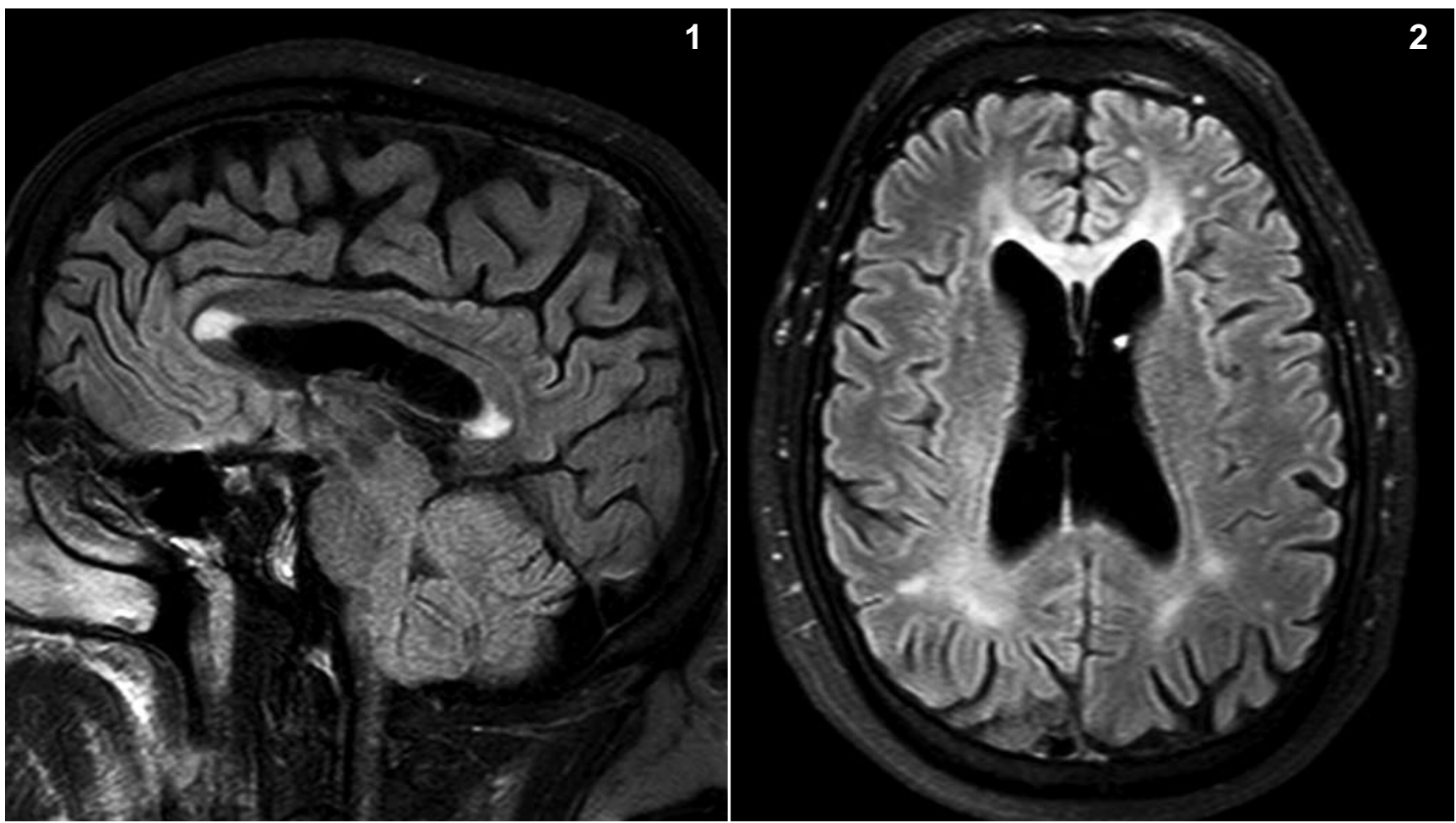

Figures: (1) Sagittal, (2) Axial, FLAIR-weighted brain MR image demonstrating anterior and posterior corpus callosal signal hyperintensities that are characteristic of MBD. Note the absence of Dawson's fingers (demyelinating lesions extending out of the corpus callosum), which are characteristic of multiple sclerosis.

shown that occupational inhalation of solvent vapors can have a deleterious effect on the nervous system. For instance, exposure to Methyl-Ethyl Ketone, may cause behavioral changes, such as emotional lability, low stress tolerance, and a tendency of hyper reactivity to conflict. ${ }^{9}$ Additional features include diffuse neurological symptoms like headache, insomnia, anxiety. Exposure to acetone and cyclohexanone, on the other hand, may lead to decreased attention span and delayed reaction time. ${ }^{10}$ To date, there is no evidence to suggest that MBD may be caused by inhalation of solvent vapors but such a possibility cannot be completely excluded.

Pathologically, the presence of symmetric demyelination and necrosis of the central part of the corpus callosum, with relative sparing of thin upper and lower edges, is characteristic of MBD. ${ }^{11}$ The entire corpus callosum may be involved. Other white matter tracts, such as the anterior and posterior commissures and the cortico-spinal tracts may also be involved. The corpus callosum degenerates and splits into three layers, termed "layered necrosis", leading to the formation of cystic cavities. ${ }^{8}$ Marchiafava-Bignami disease needs to be differentiated from other diseases affecting the corpus callosum such as ischemic stroke, contusion, multiple sclerosis (MS), paraneoplastic antibodies, and lymphoma. Marchiafava-Bignami disease is distinguished from these disorders by the symmetry of the callosal lesions, selective involvement of the entire length of the corpus callosum and focal cystic necrosis confined to its central layer. In addition to these callosal lesions, patients may also have a cerebral cortical lesion called Morel's laminar sclerosis. It is characterized by cortical laminar necrosis and gliosis, mainly in the third layer of the lateral-frontal cortex, and is probably secondary to the callosal lesions of MBD. ${ }^{11}$

On the MRI, there is diffuse atrophy of the corpus callosum, with areas of focal necrosis that are seen as hypointensities on T1 and hyperintensities on T2 weighted imaging. ${ }^{12}$ This patient did have selective involvement of the corpus callosum in the form of anterior and posterior hyperintensities on FLAIR-weighted images. That is a rare finding. Other demyelinating diseases, like MS, usually affect the mid-corpus callosum and cause "Dawson's Fingers". In addition, MS is less common in middleaged males. ${ }^{13}$ Finally, our patient did not have the classic periventricular white matter changes and did not have CSF oligoclonal bands, elevated CSF protein, IgG synthesis, or IgG index.

Ischemic disease was another possibility. However, the anterior and posterior corpus callosum are fed by different arteries and it seems unlikely they would be involved coincidentally in the absence of other signs of ischemic disease. ${ }^{14}$ We also considered CNS lymphoma, which can present with multiple focal lesions and can involve the corpus callosum. But several other features suggested that CNS lymphoma was less likely. He had no other changes in immune markers, absence of a characteristic dramatic response to 
steroids, and following him for another year has not lead to any other manifestations of lymphoma. ${ }^{15}$ Finally, a Wernicke's encephalopathy-spectrum disorder was ruled-out: the patient did not have a history of alcoholism or nutritional deficiency, neurological abnormalities such as ophthalmoplegia and ataxia, or lesions in the medial thalamus, mammillary bodies, and periaqueductal brain stem, which are usually present in Wernicke's encephalopathy. ${ }^{16}$

There is no established treatment for MBD. In the past, reports have suggested that high doses of intravenous vitamins ${ }^{7}$ or corticosteroid administration ${ }^{6}$ can be efficacious. The patients who benefited from these treatments were either alcoholic or malnourished or both, providing a rationale for the treatment. A role for these regimens in the absence of a history of alcoholism, or clinical and laboratory evidence of malnutrition, is unclear. Nonetheless, we treated our patient with a course of high doses of oral vitamin B-complex and then a short course of intravenous steroids followed by 4-Amino Pyridine (4-AP). While he was refractory to the B-complex treatment, he showed improvement with steroids and 4-AP. Interestingly, this phase did not last long and he returned to baseline. The prognosis for our patient is unclear since there are very few reported cases of MBD in nonalcoholic patients.

Because it is uncommon, MBD may be underdiagnosed or it could be misdiagnosed as another entity associated with chronic alcoholism. However, it should be considered in patients presenting with encephalopathy, motor findings, and evidence of demyelination on MRI. Intravenous vitamins and/or corticosteroid treatment should be considered in alcoholics, but it is unclear whether they will benefit non-alcoholic MDB patients.

\section{REFERENCES}

1. Kohler CG, Ances BM, Coleman AR, Ragland JD, Lazarev M, Gur RC. Marchiafava-Bignami disease: literature review and case report. Neuropsychiatry Neuropsychol Behav Neurol. 2000;13 (1):67-76.

2. Helenius J, Tatlisumak T, Soinne L, Valanne L, Kaste M. Marchiafava-Bignami disease: two cases with favourable outcome. Eur J Neurol. 2001;8(3):269-72.

3. Heinrich A, Runge U, Khaw AV. Clinicoradiologic subtypes of Marchiafava-Bignami disease. J Neurol. 2004;251(9):1050-9.

4. Rickert CH, Karger B, Varchmin-Schultheiss K, Brinkmann B, Paulus W. Neglect-associated fatal Marchiafava-Bignami disease in a non-alcoholic woman. Int J Legal Med. 2001;115(2): 90-3.

5. Celik Y, Temizoz O, Genchellac H, Cakir B, Asil T. A non-alcoholic patient with acute Marchiafava-Bignami disease associated with gynecologic malignancy: paraneoplastic marchiafava-bignami disease? Clin Neurol Neurosurg. 2007;109(6):505-8.

6. Tao H, Kitagawa N, Kako Y, Yamanaka H, Ito K, Denda K, et al. A case of anorexia nervosa with Marchiafava-Bignami disease that responded to high-dose intravenous corticosteroid administration. Psychiatry Res. 2007;156(2):181-4.

7. Tobita M, Mochizuki H, Takahashi S, Onodera H, Itoyama Y, Iwasaki Y. A case of Marchiafava-Bignami disease with complete recovery: sequential imaging documenting improvement of callosal lesions. Tohoku J Exp Med. 1997;182 (2): $175-9$.

8. Arbelaez A, Pajon A, Castillo M. Acute marchiafava-bignami disease: MR findings in two patients. AJNR Am J Neuroradiol. 2003;24(10):1955-7.

9. LoSasso GL, Rapport LJ, Axelrod BN. Neuropsychological symptoms associated with low-level exposure to solvents and (meth)acrylates among nail technicians. Neuropsychiatry Neuropsychol Behav Neurol. 2001;14(3):183-9.
10. Mitran E, Callender T, Orha B, Dragnea P, Botezatu G. Neurotoxicity associated with occupational exposure to acetone, methyl ethyl ketone, and cyclohexanone. Environ Res. 1997;73 (1-2):181-8.

11. Johkura K, Naito M, Naka T. Cortical involvement in MarchiafavaBignami disease. AJNR Am J Neuroradiol. 2005 Mar;26(3): 670-3.

12. Chang KH, Cha SH, Han MH, Park SH, Nah DL, Hong JH. Marchiafava-Bignami disease: serial changes in corpus callosum on MRI. Neuroradiology. 1992;34(6):480-2.

13. Gean-Marton AD, Vezina LG, Marton KI, Stimac GK, Peyster RG, Taveras JM, et al. Abnormal corpus callosum: a sensitive and specific indicator of multiple sclerosis. Radiology. 1991;180: 215-21.

14. Chrysikopoulos H, Andreou J, Roussakis A, Pappas J. Infarction of the corpus callosum: computed tomography and magnetic resonance imaging. Eur J Radiol. 1997;25(1):2-8.

15. Johnson BA, Fram EK, Johnson PC, Jacobowitz R. The variable MR appearance of primary lymphoma of the central nervous system: comparison with histologic features. AJNR Am J Neuroradiol. 1997;18(3):563-72.

16. Bourekas EC, Varakis K, Bruns D, Christoforidis GA, Baujan M, Slone HW, et al. Lesions of the corpus callosum: MR imaging and differential considerations in adults and children. AJR Am J Roentgenol. 2002;179(1):251-7. 